\title{
Models for the 3D singular isotropic oscillator quadratic algebra
}

\author{
E. G. Kalnins ${ }^{1}$ W. Miller, Jr., ${ }^{2}$ and S. Post ${ }^{2}$ \\ ${ }^{1}$ Department of Mathematics, University of Waikato, Hamilton, New Zealand. \\ ${ }^{2}$ School of Mathematics, University of Minnesota, Minneapolis, Minnesota, U.S.A.
}

We give the first explicit construction of the quadratic algebra for a 3D quantum superintegrable system with nondegenerate (4-parameter) potential together with realizations of irreducible representations of the quadratic algebra in terms of differential-differential or differential-difference and difference-difference operators in two variables. The example is the singular isotropic oscillator. We point out that the quantum models arise naturally from models of the Poisson algebras for the corresponding classical superintegrable system. These techniques extend to quadratic algebras for superintegrable systems in $n$ dimensions and are closely related to Hecke algebras and multivariable orthogonal polynomials.

PACS numbers: 02.00.00, 02.20.Qs,02.30.Ik, 03.65.Fd

\section{INTRODUCTION}

The distinct classical and quantum second order superintegrable systems on real or complex 3D flat space and with nondegenerate (4-parameter) potentials have been classified [1]. (Recall that a second order superintegrable system in $n$ dimensions is one that admits $2 n-1$ functionally independent constants of the motion quadratic in the momentum variables, the maximum possible, [2-4].) Indeed, the classification for nondegenerate potentials on 3D conformally flat spaces is virtually complete, [5-9]. Characteristic of these systems in all dimensions is that the second order constants of the motion generate a finite dimensional algebra, polynomially closed under commutation, the quadratic algebra. In several recent papers $[10,11]$ for the $2 \mathrm{D}$ cases, the authors have launched a study of the irreducible representations of these algebras and their applications via models of the representations, in terms of differential and difference operators. (Some earlier work on this subject can be found in [13-18].) For the 3D case where $2 \mathrm{n}-1=5$, we have shown that in fact there are always 6 linearly independent second order symmetries and that these generate a quadratic 
algebra closing at order 6 in the momenta. The second order generators are always functionally dependent via a polynomial relation at order 8. In this paper we present, for the first time, the details for a nontrivial quadratic algebra in 3D: the singular isotropic oscillator. Two-variable models for irreducible representations of the quantum system follow directly from models for the classical system. There are three possible models expressed in differential or difference operators, corresponding to separation of the eigenvalue equation for the Schrödinger operator in Cartesian, cylindrical or spherical coordinates. The models have important connections with the theory of dual Hahn and Wilson polynomials. This is a prototype for the general study of the representations of 3D quadratic algebras.

\section{THE QUANTUM SUPERINTEGRABLE SYSTEM}

The Hamiltonian operator is

$$
H=\partial_{1}^{2}+\partial_{2}^{2}+\partial_{3}^{2}+a^{2}\left(x_{1}^{2}+x_{2}^{2}+x_{3}^{2}\right)+\frac{b_{1}}{x_{1}^{2}}+\frac{b_{2}}{x_{2}^{2}}+\frac{b_{3}}{x_{3}^{2}} \quad \partial_{i} \equiv \partial_{x_{i}} .
$$

A basis for the second order constants of the motion is (with $H=M_{1}+M_{2}+M_{3}$.)

$$
M_{\ell}=\partial_{\ell}^{2}+a^{2} x_{\ell}^{2}+\frac{b_{\ell}}{x_{\ell}^{2}}, \quad \ell=1,2,3, L_{i}=\left(x_{j} \partial_{k}-x_{k} \partial_{j}\right)^{2}+\frac{b_{j} x_{k}^{2}}{x_{j}^{2}}+\frac{b_{k} x_{j}^{2}}{x_{k}^{2}},
$$

where $i, j, k$ are pairwise distinct and run from 1 to 3 . There are 4 linearly independent commutators of the second order symmetries:

$$
\begin{gathered}
S_{1}=\left[L_{1}, M_{2}\right]=\left[M_{3}, L_{1}\right], S_{2}=-\left[M_{3}, L_{2}\right]=\left[M_{1}, L_{2}\right], \\
S_{3}=-\left[M_{1}, L_{3}\right]=\left[M_{2}, L_{3}\right], R=\left[L_{1}, L_{2}\right]=\left[L_{2}, L_{3}\right]=\left[L_{3}, L_{1}\right], \\
{\left[M_{i}, M_{j}\right]=\left[M_{i}, L_{i}\right]=0, \quad 1 \leq i, j \leq 3 .}
\end{gathered}
$$

Here we define the commutator of linear operators $F, G$ by $[F, G]=F G-G F$. (Thus a second order constant of the motion is a second order partial differential operator $K$ in the variables $x_{j}$ such that $[K, H]=0$, where 0 is the zero operator.)

The fourth order structure equations are $\left[M_{i}, S_{i}\right]=0,1=1,2,3$, and

$$
\begin{gathered}
\epsilon_{i j k}\left[M_{i}, S_{j}\right]=8 M_{i} M_{k}-16 a^{2} L_{j}+8 a^{2}, \epsilon_{i j k}\left[M_{i}, R\right]=8\left(M_{j} L_{j}-M_{k} L_{k}\right)+4\left(M_{k}-M_{j}\right), \\
\epsilon_{i j k}\left[S_{i}, L_{j}\right]=8 M_{i} L_{i}-8 M_{k} L_{k}+4\left(M_{k}-M_{i}\right),
\end{gathered}
$$




$$
\begin{gathered}
\epsilon_{i j k}\left[L_{i}, S_{i}\right]=4\left\{L_{i}, M_{k}-M_{j}\right\}+16 b_{j} M_{k}-16 b_{k} M_{j}+8\left(M_{k}-M_{j}\right), \\
\epsilon_{i j k}\left[L_{i}, R\right]=4\left\{L_{i}, L_{k}-L_{j}\right\}-16 b_{j} L_{j}+16 b_{k} L_{k}+8\left(L_{k}-L_{j}+b_{j}-b_{k}\right) .
\end{gathered}
$$

Here, $\{F, G\}=F G+G F$ and $\epsilon_{i j k}$ is the completely antisymmetric tensor.

The fifth order structure equations are obtainable directly from the fourth order equations and the Jacobi identity. The sixth order structure equations are

$$
\begin{gathered}
S_{i}^{2}-\frac{8}{3}\left\{L_{j}, M_{j}, M_{k}\right\}+16 a^{2} L_{i}^{2}+\left(16 b_{k}+12\right) M_{j}^{2}+\left(16 b_{j}+12\right) M_{k}^{2}-\frac{104}{3} M_{j} M_{k} \\
-\frac{176}{3} a^{2} L_{i}-\frac{16}{3} a^{2}\left(2+9 b_{j}+9 b_{k}+12 b_{j} b_{k}\right)=0 \\
\frac{1}{2}\left\{S_{i}, S_{j}\right\}+\frac{4}{3}\left\{L_{i}, M_{k}, M_{i}\right\}+\frac{4}{3}\left\{L_{j}, M_{k}, M_{j}\right\}-8 L_{k} M_{k}^{2}-8 a^{2}\left\{L_{i}, L_{j}\right\}-\left(16 b_{k}+12\right) M_{i} M_{j} \\
+4 M_{k}^{2}-4 M_{k}\left(M_{i}+M_{j}\right)+a^{2}\left(32 b_{k}+24\right) L_{k}+8 a^{2}\left(L_{i}+L_{j}\right)-16 a^{2}\left(b_{k}+1\right)=0 \\
\frac{1}{2}\left\{S_{i}, R\right\}-8 L_{i}^{2} M_{i}+\frac{4}{3}\left\{L_{k}, L_{i}, M_{k}\right\}+\frac{4}{3}\left\{L_{i}, L_{j}, M_{j}\right\}-\left(8 b_{k}+6\right)\left\{L_{k}, M_{j}\right\} \\
-\left(8 b_{j}+6\right)\left\{L_{j}, M_{k}\right\}-2\left\{L_{i}, M_{k}+M_{j}\right\}+\frac{88}{3} L_{i} M_{i}+\frac{52}{3}\left(L_{k} M_{k}+L_{j} M_{j}\right) \\
+\left(32 b_{k} b_{j}+24 b_{k}+24 b_{j}+\frac{16}{3}\right) M_{i}+\left(8 b_{j}-\frac{8}{3}\right) M_{k}+\left(8 b_{k}-\frac{8}{3}\right) M_{j}=0 \\
R^{2}-\frac{8}{3}\left\{L_{1}, L_{2}, L_{3}\right\}+\left(16 b_{1}+12\right) L_{1}^{2}+\left(16 b_{2}+12\right) L_{2}^{2}+\left(16 b_{3}+12\right) L_{3}^{2}-\frac{52}{3}\left\{L_{1}, L_{2}\right\} \\
-\frac{52}{3}\left\{L_{1}, L_{3}\right\}-\frac{52}{3}\left\{L_{2}, L_{3}\right\}-\frac{16}{3}\left(11 b_{1}+1\right) L_{1}-\frac{16}{3}\left(11 b_{2}+1\right) L_{2} \\
-\frac{16}{3}\left(11 b_{3}+1\right) L_{3}-\frac{32}{3}\left(6 b_{1} b_{2} b_{3}+\frac{9}{2}\left(b_{1} b_{2}+b_{1} b_{3}+b_{2} b_{3}\right)+b_{1}+b_{2}+b_{3}\right) .
\end{gathered}
$$

Here, $\{A, B, C\}=A B C+A C B+B A C+B C A+C A B+C B A$ and $i, j, k$ are pairwise distinct.

The eighth order functional relation is

$$
\begin{gathered}
L_{1}^{2} M_{1}^{2}+L_{2}^{2} M_{2}^{2}+L_{3}^{2} M_{3}^{2}-\frac{1}{12}\left\{L_{1}, L_{2}, M_{1}, M_{2}\right\}-\frac{1}{12}\left\{L_{1}, L_{3}, M_{1}, M_{3}\right\} \\
-\frac{1}{12}\left\{L_{2}, L_{3}, M_{2}, M_{3}\right\}-\frac{7}{3} L_{1} M_{1}^{2}-\frac{7}{3} L_{2} M_{2}^{2}-\frac{7}{3} L_{3} M_{3}^{2}+\frac{2}{3} a\left\{L_{1}, L_{2}, L_{3}\right\} \\
-\frac{1}{18}\left\{L_{1}, M_{1}, M_{2}\right\}-\frac{1}{18}\left\{L_{1}, M_{1}, M_{3}\right\}-\frac{1}{18}\left\{L_{2}, M_{1}, M_{2}\right\}-\frac{1}{18}\left\{L_{2}, M_{2}, M_{3}\right\} \\
-\frac{1}{18}\left\{L_{3}, M_{1}, M_{3}\right\}-\frac{1}{18}\left\{L_{3}, M_{2}, M_{3}\right\}+\frac{1}{6}\left(4 b_{1}+3\right)\left\{L_{1}, M_{2}, M_{3}\right\} \\
+\frac{1}{6}\left(4 b_{2}+3\right)\left\{L_{2}, M_{1}, M_{3}\right\}+\frac{1}{6}\left(4 b_{3}+3\right)\left\{L_{3}, M_{1}, M_{2}\right\}-a^{2}\left(4 b_{1}+3\right) L_{1}^{2}-a^{2}\left(4 b_{2}+3\right) L_{2}^{2}-a^{2}\left(4 b_{3}+3\right) L_{3}^{2}
\end{gathered}
$$




$$
\begin{gathered}
+\frac{a^{2}}{3}\left(\left\{L_{1}, L_{2}\right\}+\left\{L_{1}, L_{3}\right\}+\left\{L_{2}, L_{3}\right\}\right)-\left(4 b_{2} b_{3}+3 b_{2}+3 b_{3}+\frac{4}{3}\right) M_{1}^{2} \\
-\left(4 b_{1} b_{3}+3 b_{1}+3 b_{3}+\frac{4}{3}\right) M_{2}^{2}-\left(4 b_{1} b_{2}+3 b_{1}+3 b_{2}+\frac{4}{3}\right) M_{3}^{2}+\frac{2}{3}\left(b_{3}+2\right) M_{1} M_{2} \\
+\frac{2}{3}\left(b_{2}+2\right) M_{1} M_{3}+\frac{2}{3}\left(b_{1}+2\right) M_{2} M_{3}+\frac{4}{3} a^{2}\left(7 b_{1}+4\right) L_{1}+\frac{4}{3} a^{2}\left(7 b_{2}+4\right) L_{2} \\
+\frac{4}{3} a^{2}\left(7 b_{3}+4\right) L_{3}+\frac{4}{3} a^{2}\left(12 b_{1} b_{2} b_{3}+9 b_{1} b_{2}+9 b_{1} b_{3}+9 b_{2} b_{3}+4 b_{1}+4 b_{2}+4 b_{3}\right)=0 .
\end{gathered}
$$

Here, $\{A, B, C, D\}$ is the 24 term symmetrizer of 4 operators.

\section{A. Cartesian case: A quantum model with $M_{1}, M_{2}$ diagonal}

For the model we choose variables $u, v$ in which the eigenfunctions are polynomials, and write the parameters as $b_{j}=1 / 4-k_{j}^{2}$. Then we have

$$
\begin{gathered}
M_{1}=2 i a\left(2 u \partial_{u}+k_{1}+1\right), M_{2}=2 i a\left(2 v \partial_{v}+k_{2}+1\right), M_{1}+M_{2}+M_{3}=E \\
L_{1}=4 v\left(u^{2} \partial_{u}^{2}+2 u v \partial_{u} \partial_{v}+\left(v^{2}+1\right) \partial_{v}^{2}-\left(\frac{E}{2 i a}-k_{1}-k_{2}-4\right) u \partial_{u}-\left(\frac{E}{2 i a}-k_{1}-k_{2}-4\right) \partial_{v}\right) \\
+4\left(1+k_{2}\right) \partial_{v}+v\left(\left(-\frac{E}{2 i a}+k_{1}+k_{2}+3\right)^{2}-k_{3}^{2}\right)+\frac{1}{2 a^{2}} M_{2} M_{3}+\frac{1}{2} \\
L_{2}=4 u\left(v^{2} \partial_{v}^{2}+2 u v \partial_{u} \partial_{v}+\left(u^{2}+1\right) \partial_{u}^{2}-\left(\frac{E}{2 i a}-k_{1}-k_{2}-4\right) v \partial_{v}-\left(\frac{E}{2 i a}-k_{1}-k_{2}-4\right) u \partial_{u}\right) \\
+4\left(1+k_{1}\right) \partial_{u}+u\left(\left(-\frac{E}{2 i a}+k_{1}+k_{2}+3\right)^{2}-k_{3}^{2}\right)+\frac{1}{2 a^{2}} M_{1} M_{3}+\frac{1}{2} \\
L_{3}=4\left(u v \partial_{u}^{2}+u v \partial_{v}^{2}+\left(k_{1}+1\right) v \partial_{u}+\left(k_{2}+1\right) u \partial_{v}\right)+\frac{1}{2 a^{2}} M_{1} M_{2}+\frac{1}{2} .
\end{gathered}
$$

In the model, the monomials $f_{N, j}=u^{j} v^{N-j}$ are simultaneous eigenfunctions of the operators $M_{j}$ :

$$
M_{1} f_{N, j}=2 i a\left(2 j+k_{1}+1\right) f_{N, j}, M_{2} f_{N, j}=2 i a\left(2 N-2 j+k_{2}+1\right) f_{N, j}
$$

Further, we have the expansion formulas

$$
\begin{aligned}
& L_{1} f_{N, j}=\left(\left(2 N+3-\frac{E}{2 i a}+k_{1}+k_{2}\right)^{2}-k_{3}^{2}\right) f_{N+1, j}+4(N-j)\left(N-j+k_{2}\right) f_{N-1, j} \\
& +\left(2\left(\frac{E}{2 i a}-2 N-2 k_{1}-2 k_{2}-2\right)\left(2 N-2 j+k_{2}+1\right)+\frac{1}{2}\right) f_{N, j}
\end{aligned}
$$




$$
\begin{gathered}
L_{2} f_{N, j}=\left(\left(2 N+3-\frac{E}{2 i a}+k_{1}+k_{2}\right)^{2}-k_{3}^{2}\right) f_{N+1, j+1}+4 j\left(j+k_{1}\right) f_{N-1, j-1} \\
+\left(2\left(\frac{E}{2 i a}-2 N-2 k_{1}-2 k_{2}-2\right)\left(2 j+k_{1}+1\right)+\frac{1}{2}\right) f_{N, j} \\
L_{3} f_{N, j}=4(N-j)\left(N-j+k_{2}\right) f_{N, j+1}+4 j\left(j+k_{2}\right) f_{N, j-1} \\
+\left(2\left(2 j+k_{1}+1\right)\left(2 N-2 j+k_{2}+1\right)+\frac{1}{2}\right) f_{N, j}
\end{gathered}
$$

From the model we can find a family of finite dimensional irreducible representations, labeled by the nonnegative integer $M$. A basis of eigenfunctions is given by $\left\{f_{N, j}=u^{j} v^{N-j}\right\}$, such that the $N$ and $j$ are nonnegative integers satisfying $0 \leq j \leq N \leq M$.. The energy satisfies

$$
E=2 i a\left(2 M+k_{1}+k_{2}+k_{3}+3\right)
$$

The dimension of the representation is $(M+2)(M+1) / 2$. Now we introduce an inner product such that the operators $M_{j}, L_{j}$ are formally self-adjoint for $j=1,2,3$. This forces $a$ to be pure imaginary.

Normalization coefficients: Let $\hat{f}_{N, j}=K_{N, j} u^{j} v^{N-j}$ such that $\left\|\hat{f}_{N, j}\right\|=1$. If we assume $K_{0,0}=1$ then the coefficients become,

$$
K_{N, j}=\frac{(-M)_{N}\left(-M-k_{3}\right)_{N}}{(N-j) ! j !\left(k_{2}+1\right)_{N-j}\left(k_{1}+1\right)_{j}}
$$

\section{B. Recurrence relations for Wilson polynomials}

The spherical case is is intimately bound up with recurrence relations for Wilson polynomials and the cylindrical case with the dual Hahn polynomials. To see this we modify some of the results of [19]. The unnormalized Wilson polynomials are

$$
\begin{gathered}
w_{n}\left(y^{2}\right) \equiv w_{n}\left(y^{2}, \alpha, \beta, \gamma, \delta\right)=(\alpha+\beta)_{n}(\alpha+\gamma)_{n}(\alpha+\delta)_{n} \times \\
{ }_{4} F_{3}\left(\begin{array}{cc}
-n, & \alpha+\beta+\gamma+\delta+n-1, \alpha-y, \alpha+y \\
\alpha+\beta, \alpha+\gamma, & \alpha+\delta
\end{array}\right) \\
=(\alpha+\beta)_{n}(\alpha+\gamma)_{n}(\alpha+\delta)_{n} \Phi_{n}^{(\alpha, \beta, \gamma, \delta)}\left(y^{2}\right),
\end{gathered}
$$

where $(a)_{n}$ is the Pochhammer symbol and ${ }_{4} F_{3}(1)$ is a generalized hypergeometric function of unit argument. 
For fixed $\alpha, \beta, \gamma, \delta>0$ the Wilson polynomials are orthogonal with respect to the inner product

$$
\begin{gathered}
<w_{n}, w_{n^{\prime}}>=\frac{1}{2 \pi} \int_{0}^{\infty} w_{n}\left(-y^{2}\right) w_{n^{\prime}}\left(-y^{2}\right)\left|\frac{\Gamma(\alpha+i y) \Gamma(\beta+i y) \Gamma(\gamma+i y) \Gamma(\delta+i y)}{\Gamma(2 i y)}\right|^{2} d y \\
=\delta_{n n^{\prime}} n !(\alpha+\beta+\gamma+\delta+n-1)_{n} \times \\
\frac{\Gamma(\alpha+\beta+n) \Gamma(\alpha+\gamma+n) \Gamma(\alpha+\delta+n) \Gamma(\beta+\gamma+n) \Gamma(\beta+\delta+n) \Gamma(\gamma+\delta+n)}{\Gamma(\alpha+\beta+\gamma+\delta+2 n)} .
\end{gathered}
$$

The Wilson polynomials $\Phi_{n}\left(y^{2}\right) \equiv \Phi_{n}^{(\alpha, \beta, \gamma, \delta)}\left(y^{2}\right)$, satisfy the three term recurrence formula

$$
y^{2} \Phi_{n}\left(y^{2}\right)=K(n+1, n) \Phi_{n+1}\left(y^{2}\right)+K(n, n) \Phi_{n}\left(y^{2}\right)+K(n-1, n) \Phi_{n-1}\left(y^{2}\right)
$$

where

$$
\begin{gathered}
K(n+1, n)=\frac{\alpha+\beta+\gamma+\delta+n-1}{(\alpha+\beta+\gamma+\delta+2 n-1)(\alpha+\beta+\gamma+\delta+2 n)} \times \\
(\alpha+\beta+n)(\alpha+\gamma+n)(\alpha+\delta+n), \\
K(n-1, n)=\frac{n(\beta+\gamma+n-1)(\beta+\delta+n-1)(\gamma+\delta+n-1)}{(\alpha+\beta+\gamma+\delta+2 n-2)(\alpha+\beta+\gamma+\delta+2 n-1)}, \\
K(n, n)=\alpha^{2}-K(n+1, n)-K(n-1, n) .
\end{gathered}
$$

Moreover, they satisfy the following parameter-changing recurrence relations when acting on the basis polynomials $\Phi_{n} \equiv \Phi_{n}^{(\alpha, \beta, \gamma, \delta)}$. Here $T^{\tau} f(y)=f(y+\tau)$.

1.

$$
R=\frac{1}{2 y}\left[T^{1 / 2}-T^{-1 / 2}\right], R \Phi_{n}=\frac{n(n+\alpha+\beta+\gamma+\delta-1)}{(\alpha+\beta)(\alpha+\gamma)(\alpha+\delta)} \Phi_{n-1}^{(\alpha+1 / 2, \beta+1 / 2, \gamma+1 / 2, \delta+1 / 2)} .
$$

2.

$$
\begin{gathered}
L=\frac{1}{2 y}\left[\left(\alpha-\frac{1}{2}+y\right)\left(\beta-\frac{1}{2}+y\right)\left(\gamma-\frac{1}{2}+y\right)\left(\delta-\frac{1}{2}+y\right) T^{1 / 2}\right. \\
\left.-\left(\alpha-\frac{1}{2}-y\right)\left(\beta-\frac{1}{2}-y\right)\left(\gamma-\frac{1}{2}-y\right)\left(\delta-\frac{1}{2}-y\right) T^{-1 / 2}\right] . \\
L \Phi_{n}=(\alpha+\beta-1)(\alpha+\gamma-1)(\alpha+\delta-1) \Phi_{n+1}^{(\alpha-1 / 2, \beta-1 / 2, \gamma-1 / 2, \delta-1 / 2)} .
\end{gathered}
$$

3.

$$
\begin{gathered}
L_{\alpha \beta}=\frac{1}{2 y}\left[-\left(\alpha-\frac{1}{2}+y\right)\left(\beta-\frac{1}{2}+y\right) T^{1 / 2}+\left(\alpha-\frac{1}{2}-y\right)\left(\beta-\frac{1}{2}-y\right) T^{-1 / 2}\right] . \\
L_{\alpha \beta} \Phi_{n}=-(\alpha+\beta-1) \Phi_{n}^{(\alpha-1 / 2, \beta-1 / 2, \gamma+1 / 2, \delta+1 / 2)} .
\end{gathered}
$$


4.

$$
\begin{gathered}
R^{\alpha \beta}=\frac{1}{2 y}\left[-\left(\gamma-\frac{1}{2}+y\right)\left(\delta-\frac{1}{2}+y\right) T^{1 / 2}+\left(\gamma-\frac{1}{2}-y\right)\left(\delta-\frac{1}{2}-y\right) T^{-1 / 2}\right] . \\
R^{\alpha \beta} \Phi_{n}=-\frac{(n+\gamma+\delta-1)(n+\alpha+\beta)}{\alpha+\beta} \Phi_{n}^{(\alpha+1 / 2, \beta+1 / 2, \gamma-1 / 2, \delta-1 / 2)} .
\end{gathered}
$$

The operators $L_{\alpha \gamma}, L_{\alpha \delta}, R_{\alpha \gamma}, R_{\alpha \delta}$ are obtained by obvious substitutions.

\section{Recurrence relations for dual Hahn polynomials}

The dual Hahn polynomials are given by the formula

$$
\begin{gathered}
h_{n}\left(y^{2}\right) \equiv h_{n}\left(y^{2}, \alpha, \beta, \gamma\right)=(\alpha+\beta)_{n}(\alpha+\gamma)_{n 3} F_{2}\left(\begin{array}{cc}
-n, & \alpha-y, \alpha+y \\
\alpha+\beta, \alpha+\gamma & \alpha
\end{array}\right) \\
=(\alpha+\beta)_{n}(\alpha+\gamma)_{n} \Omega_{n}^{(\alpha, \beta, \gamma)}\left(y^{2}\right),
\end{gathered}
$$

Note that the discrete dual Hahn polynomials [20] are $p_{j}\left(y^{2}\right)=\Omega^{(-Q / 2,(A+1) / 2,(B+1) / 2)}\left(y^{2}\right)$ in this notation. The dual Hahn polynomials are obtained from the Wilson polynomials by letting $\delta \rightarrow \infty$. Indeed

$$
h_{n}\left(y^{2}, \alpha, \beta, \gamma\right)=\lim _{\delta \rightarrow \infty} \frac{w_{n}\left(y^{2}, \alpha, \beta, \gamma, \delta\right)}{(\alpha+\delta)_{n}}, \quad \Omega_{n}^{(\alpha, \beta, \gamma)}\left(y^{2}\right)=\lim _{\delta \rightarrow \infty} \Phi_{n}^{(\alpha, \beta, \gamma, \delta)}\left(y^{2}\right) .
$$

It follows immediately that $h_{n}\left(y^{2}, \alpha, \beta, \gamma\right)$ is symmetric in $\alpha, \beta, \gamma$.

The recurrence relations for Wilson polynomials presented in the previous section go in the limit to parameter changing recurrences for dual Hahn polynomials. The three term recurrence relation for the dual Hahn polynomials is

$$
\left(-\alpha^{2}+y^{2}\right) \Omega_{n}\left(y^{2}\right)=K(n+1, n) \Omega_{n+1}\left(y^{2}\right)+K(n, n) \Omega_{n}\left(y^{2}\right)+K(n-1, n) \Omega_{n-1}\left(y^{2}\right)
$$

where $K(n+1, n)=(n+\alpha+\beta)(n+\alpha+\gamma), K(n-1, n)=n(n+\beta+\gamma-1), K(n, n)=$ $-K(n+1, n)-K(n-1, n)$. There are 8 basic raising and lowering operators for the dual Hahn polynomials. We list them here and describe their actions on the basis polynomials $\Omega_{n} \equiv \Omega_{n}^{(\alpha, \beta, \gamma)}$.

1.

$$
R=\frac{1}{2 y}\left[T^{1 / 2}-T^{-1 / 2}\right], R \Omega_{n}=\frac{n}{(\alpha+\beta)(\alpha+\gamma)} \Omega_{n-1}^{(\alpha+1 / 2, \beta+1 / 2, \gamma+1 / 2)}
$$


2.

$$
\begin{gathered}
L=\frac{1}{2 y}\left[P\left(y-\frac{1}{2}\right) T^{1 / 2}-P\left(-y-\frac{1}{2}\right) T^{-1 / 2}\right] . \\
P(y)=(\alpha+y)(\beta+y)(\gamma+y), L \Omega_{n}=(\alpha+\beta-1)(\alpha+\gamma-1) \Omega_{n+1}^{(\alpha-1 / 2, \beta-1 / 2, \gamma-1 / 2)} .
\end{gathered}
$$

3.

$$
\begin{gathered}
R^{\alpha}=\frac{1}{2 y}\left[-\left(\beta+y-\frac{1}{2}\right)\left(\gamma+y-\frac{1}{2}\right) T^{1 / 2}+\left(\beta-y-\frac{1}{2}\right)\left(\gamma-y-\frac{1}{2}\right) T^{-1 / 2}\right] \\
R^{\alpha} \Omega_{n}=-(\beta+\gamma+n-1) \Omega_{n}^{(\alpha+1 / 2, \beta-1 / 2, \gamma-1 / 2)}
\end{gathered}
$$

4.

$$
\begin{gathered}
R^{\beta}=\frac{1}{2 y}\left[-\left(\alpha+y-\frac{1}{2}\right)\left(\gamma+y-\frac{1}{2}\right) T^{1 / 2}+\left(\alpha-y-\frac{1}{2}\right)\left(\gamma-y-\frac{1}{2}\right) T^{-1 / 2}\right] \\
R^{\beta} \Omega_{n}=-(\alpha+\gamma-1) \Omega_{n}^{(\alpha-1 / 2, \beta+1 / 2, \gamma-1 / 2)}
\end{gathered}
$$

5.

$$
\begin{gathered}
R^{\gamma}=\frac{1}{2 y}\left[-\left(\alpha+y-\frac{1}{2}\right)\left(\beta+y-\frac{1}{2}\right) T^{1 / 2}+\left(\alpha-y-\frac{1}{2}\right)\left(\beta-y-\frac{1}{2}\right) T^{-1 / 2}\right] . \\
R^{\gamma} \Omega_{n}=-(\alpha+\beta-1) \Omega_{n}^{(\alpha-1 / 2, \beta-1 / 2, \gamma+1 / 2)}
\end{gathered}
$$

6.

$$
L_{\alpha}=\frac{1}{2 y}\left[-\left(\alpha-\frac{1}{2}+y\right) T^{1 / 2}+\left(\alpha-\frac{1}{2}-y\right) T^{-1 / 2}\right], L_{\alpha} \Omega_{n}=-\Omega_{n}^{(\alpha-1 / 2, \beta+1 / 2, \gamma+1 / 2)}
$$

7.

$$
\begin{gathered}
L_{\beta}=\frac{1}{2 y}\left[-\left(\beta-\frac{1}{2}+y\right) T^{1 / 2}+\left(\beta-\frac{1}{2}-y\right) T^{-1 / 2}\right], \\
L_{\beta} \Omega_{n}=-\left(\frac{n+\alpha+\gamma}{\alpha+\gamma}\right) \Omega_{n}^{(\alpha+1 / 2, \beta-1 / 2, \gamma+1 / 2)}
\end{gathered}
$$

8.

$$
\begin{gathered}
L_{\gamma}=\frac{1}{2 y}\left[-\left(\gamma-\frac{1}{2}+y\right) T^{1 / 2}+\left(\gamma-\frac{1}{2}-y\right) T^{-1 / 2}\right], \\
L_{\gamma} \Omega_{n}=-\left(\frac{n+\alpha+\beta}{\alpha+\beta}\right) \Omega_{n}^{(\alpha+1 / 2, \beta+1 / 2, \gamma-1 / 2)}
\end{gathered}
$$




\section{Cylindrical case: A quantum model with $L_{3}, M_{3}$ diagonal}

For the model of the finite dimensional irreducible representation (15) the basis eigenfunctions of $M_{1}$ and $M_{2}$ have the form $\psi_{N, j}=d_{N, j} t^{N} \delta\left(y-\lambda_{j}\right), \lambda_{j}=j+\frac{k_{1}+k_{2}+1}{2}$. We use the differential operator $\partial_{t}$ and the difference operator $T^{\tau}(f(y, t))=f(y+\tau, t)$ to construct our model operators. Setting,

$$
\alpha=-N-\frac{k_{1}+k_{2}+1}{2} \quad \beta=\frac{k_{1}+k_{2}+1}{2} \quad \gamma=\frac{k_{1}-k_{2}+1}{2},
$$

we have the model

$$
\begin{gathered}
M_{1}=2 i a\left(2 \mathbf{L} R-2 t \partial_{t}+\beta-\gamma\right) \\
M_{3}=2 i a\left(2 t \partial_{t}-2 M-k_{3}+1\right) \\
M_{1}+M_{2}+M_{3}=E \\
L_{1}=\frac{1}{2}\left(\frac{1}{t} R^{\alpha} R+8 t\left(M-t \partial_{t}\right)\left(M-t \partial_{t}-k_{3}\right) \mathbf{L}_{\alpha} \mathbf{L}+\frac{1}{2 \alpha^{2}} M_{2} M_{3}+\frac{1}{2}\right) \\
L_{2}=\frac{1}{2}\left(\frac{1}{t} L_{\beta} L_{\gamma}+8 t\left(M-t \partial_{t}\right)\left(M-t \partial_{t}-k_{3}\right) \mathbf{R}^{\beta} \mathbf{R}^{\gamma}+\frac{1}{2 \alpha^{2}} M_{1} M_{3}+\frac{1}{2}\right) \\
L_{3}=-4 y^{2}+k_{1}^{2}+k_{2}^{2}
\end{gathered}
$$

Here $R, L, R^{\alpha}, \cdots$ are the difference operators for dual Hahn polynomials defined in Section IIC. An operator in bold face, e.g., $\mathbf{R}^{\beta}$ indicates that in the expression for the $y$ difference operator the parameter $\alpha=-N-\left(k_{1}+k_{2}+1\right) / 2$ is replaced by the differential operator $-t \partial_{t}-\left(k_{1}+k_{2}+1\right) / 2$. Note that the dual Hahn polynomials $\Omega_{n}^{(\alpha, \beta, \gamma)}\left(y^{2}\right) t^{N}$ are simultaneous eigenfunctions of operators $M_{1}$ and $M_{3}$ in this model.

\section{E. Spherical case: A quantum model with $L_{1}+L_{2}+L_{3}, L_{3}$ diagonal}

For the model of the finite dimensional irreducible representation (15) the basis eigenfunctions of $L_{3}$ and $L_{1}+L_{2}+L_{3}$ have the form $\phi_{N, j}=C_{N, j} \delta\left(y-\lambda_{j}\right) \delta\left(z-\lambda_{N}\right)$ where the support of the finite measure is $\lambda_{j}=j+\frac{k_{1}+k_{2}+1}{2} \quad \lambda_{N}=N+1-\frac{k_{1}+k_{2}-k_{3}}{2}$. We use the difference operators $T^{\tau}(f(y, z))=f(y+\tau, z)$ and $Z^{\tau}(f(y, z))=f(y, z+\tau)$ to construct our model operators. Setting

$$
\alpha=z+\frac{k_{3}+1}{2} \quad \delta=-z+\frac{k_{3}+1}{2} \quad \beta=\frac{k_{1}-k_{2}+1}{2} \quad \gamma=\frac{1-k_{1}-k_{2}}{2},
$$


we find the model

$$
\begin{gathered}
L_{1}=2 L R+(\alpha+\delta)(\beta+\gamma) \\
L_{3}=-4 y^{2}+2\left(\gamma^{2}-\gamma+\beta^{2}-\beta\right)+1, \\
L_{1}+L_{2}+L_{3}=4 z^{2}+k_{1}^{2}-k_{2}^{2}-k_{3}+\frac{3}{2}=-4(\alpha \delta-\gamma \beta)+2(\alpha+\delta+\gamma+\beta)-\frac{1}{2}, \\
\frac{M_{1}}{2 i \alpha}=\frac{2(\alpha+\delta-5-2 M+2 \gamma)}{(\alpha-\delta-1)(\alpha-\delta+1)}(L R+1+2 \gamma \beta-\beta-\gamma(\gamma+\beta-1)(\alpha+\delta)+2 \alpha \delta) \\
\frac{M_{3}}{2 \alpha}=\frac{\alpha^{3}+\delta^{2}-2\left(\alpha^{2}+\delta^{2}\right)(M-\gamma+2)+3\left(\alpha^{2} \delta+\alpha \delta^{2}-2 \alpha \delta\right)+(\alpha+\delta)(2 M+2 \gamma+7)-2}{(\alpha-\delta)(\alpha-\delta-1)} L_{\alpha \beta} L_{\alpha \gamma}+2 Z^{-1} \frac{2-\delta-\gamma+M}{(\alpha-\delta)(\alpha-\delta+1)} R^{\alpha \beta} R^{\alpha \gamma} \\
+2 \frac{(\alpha-1-y+1)(\alpha-\delta-1)}{(\alpha-\delta)(\alpha-\delta-1)} \\
M_{1}+M_{2}+M_{3}=E
\end{gathered}
$$

where the difference operators $L, R$, etc., are defined in section IIB. Note that the Wilson polynomials (actually Racah polynomials for finite dimensional representations) $\Phi_{n}^{(\alpha, \beta, \gamma, \delta)}\left(y^{2}\right) \delta\left(z-\lambda_{N}\right)$ are simultaneous eigenfunctions of operators $L_{1}$ and $L_{1}+L_{2}+L_{3}$ in this model.

\section{DISCUSSION AND OUTLOOK}

We exhibited, for the first time, three models of the quadratic algebra for the 3D singular isotropic oscillator superintegrable system: a differential-differential, a differential-difference and a difference-difference operator model. We have presented the final results in the simplest form possible, to show the recurrence relation structure of Wilson (Racah) and dual Hahn polynomials. The models are associated with diagonalization of the operators responsible for separation of the Schrödinger eigenvalue problem in Cartesian, cylindrical and spherical coordinates, respectively. The derivation of these results, particularly the spherical model, is complicated; complete details will appear in [21]. First of all, although the general structure of the quadratic algebra follows from general theorems, the exact structure equations (4)(6) are nontrivial to derive. A model must satisfy identically all of the quadratic algebra structure equations up to 8th order. The possibility and general form of each model follows from analysis of the Poisson algebra generated by the classical superintegrable system, just 
as in the 2D cases, [10],[11]. Then the classical model must be quantized. For example it is possible to construct a classical two variable Cartesian model for which $M_{1}=A, M_{2}=B$ and $M_{3}=E-A-B$ and

$$
\begin{gathered}
L_{1}=\frac{1}{4 \alpha^{2}}\left[\left(4 \delta \alpha-C_{2}\right) \exp \left(4 i \alpha P_{B}\right)+\left(4 \gamma \alpha-B_{2}\right) \exp \left(-4 i \alpha P_{B}\right)+2 B C\right], \\
\left.L_{2}=\frac{1}{4 \alpha^{2}}\left[\left(4 \delta \alpha-C_{2}\right) \exp \left(4 i \alpha P_{A}\right)+\left(4 \beta \alpha-A_{2}\right)\right] \exp \left(-4 i \alpha P_{A}\right)+2 A C\right], \\
\left.L_{3}=\frac{1}{4 \alpha^{2}}\left[\left(4 \beta \alpha-A_{2}\right) \exp \left(4 i \alpha\left(P_{A}-P_{B}\right)\right)+\left(4 \gamma \alpha-B_{2}\right)\right] \exp \left(-4 \alpha i\left(P_{A}-P_{B}\right)\right)+2 A B\right]
\end{gathered}
$$

with $P_{A}$ and $P_{B}$ the canonical momenta conjugate to $A$ and $B$. Here, all terms except the variables $A, B, P_{A}, P_{B}$ are constants. This model then suggests the quantum Cartesian model via the quantisation rules $A \rightarrow \partial_{A}$ and $B \rightarrow \partial_{B}$. The deep connection between algebras generated by recurrences of families of orthogonal polynomials and the hidden algebras of quantum superintegrable systems may seem somewhat surprising, since the polynomials do not appear as eigenfunctions of the original quantum Hamiltonian. Rather they appear as connection coefficients between eigenfunctions of two different sets of commuting symmetry operators.

Much remains to be done. First, there appear to be connections between our models and Hecke algebras [22-24] and these relations have yet to be explored. Also, we have exhibited only the finite dimensional irreducible representations (associated with Racah polynomials) but there are also classes of infinite dimensional irreducible representations (associated with general Wilson polynomials). Intertwining operators mapping the models to the original quantum system remain to be constructed. Other nondegenerate 3D systems remain to be studied, particularly the generic potential on the 3-sphere. In that case the model will be expressible in terms of the recurrences for a family of two-variable Wilson polynomials. There are also families of degenerate 3D superintegrable systems. These have yet to be completely classified and their symmetry algebras studied. Then the study must be extended to nD second order superintegrable systems on conformally flat spaces. The formulation of a $q$ version of superintegrable systems remains open.

[1] E. G. Kalnins, W. Miller, Jr., G. C. Williams and G. S. Pogosyan, J. Phys. A: Math Gen. 35, 4655 (2002). 
[2] N. W. Evans, Phys. Rev. A. 41, 5666 (1990).

[3] M. F. Rañada, J. Math. Phys. 38, 4165 (1997).

[4] Superintegrability in Classical and Quantum Systems, P. Tempesta, P. Winternitz, W. Miller, G. Pogosyan editors, AMS, 37, 2005.

[5] E. G. Kalnins, J. M. Kress and W. Miller, Jr., J. Math. Phys. 46, 053509 (2005).

[6] E. G. Kalnins, J. M. Kress and W. Miller, Jr., J. Math. Phys. 47, 043514 (2006).

[7] C. Daskaloyannis and Y. Tanoudis, J. Math Phys. 48, 072108 (2007)

[8] E. G. Kalnins, J. M. Kress, W. Miller, Jr. and P. Winternitz, J. Math. Phys. 44, 5811 (2003).

[9] J. M. Kress, Phys. Atomic Nuclei 70, 560 (2007).

[10] E. G. Kalnins, W, Miller, Jr. and S. Post, J. Phys. A: Math. Theor. 40, 11525 (2007).

[11] E. G. Kalnins, W, Miller, Jr. and S. Post, SIGMA 4, 008 arXiv:0801.2848 (2008).

[12] E. G. Kalnins, W, Miller, Jr. and S. Post, Models of quadratic quantum algebras and their relation to classical superintegrable systems, (submitted) (2008)

[13] D. Bonatos, C. Daskaloyannis and K. Kokkotas, Phys. Rev. A. 50, 3700 (1994).

[14] C. Daskaloyannis, J. Math. Phys. 42, 1100 (2001).

[15] Y. I. Granovskii, A. S. Zhedanov and I. M. Lutsenko, Theoret. and Math. Phys. 91, 604 (1992).

[16] C. Daskaloyannis C., J. Math. Phys 42, 1100 math-ph/0003017 (2001).

[17] C. Daskaloyannis and K. Ypsilantis, J. Math Phys. 47, 042904 (2006).

[18] C. Quesne, SIGMA 3, 067 (2007).

[19] W. Miller, Jr. SIAM J. Math. Anal. 18, 1221 (1987).

[20] G. E. Andrews, R. Askey and R. Roy, Special Functions. Encyclopedia of Mathematics and its Applications, Cambridge University Press, Cambridge, UK, 1999.

[21] S. Post, University of Minnesota Ph.D. Thesis, Second order superintegrable systems, (in preparation), 2009.

[22] S. Sahi, Raising and Lowering Operators for Askey-Wilson Polynomials, SIGMA, 3, 002, (2007).

[23] T. H. Koornwinder, The relationship between Zhedanovs algebra AW (3) and the double affine Hecke algebra in the rank one case, SIGMA, 3, 063, (2007).

[24] T. H. Koornwinder, Zhedanovs Algebra AW(3) and the Double Affine Hecke Algebra in the Rank One Case. II. The Spherical Subalgebra SIGMA, 4, 052, (2008). 